\title{
NW-G03, a related cyclic hexapeptide compound of NW-G01, produced by Streptomyces alboflavus 313
}

\author{
Zhengyan Guo ${ }^{1,2}$, Ling Shen ${ }^{2}$, Jiwen Zhang ${ }^{1}$, Hongmei Xin ${ }^{1}$, Wei Liu ${ }^{3}$, Zhiqin $\mathrm{Ji}^{1}$ and Wenjun $\mathrm{Wu}^{1}$ \\ The Journal of Antibiotics (2011) 64, 789-794; doi:10.1038/ja.2011.88; published online 26 October 2011 \\ Keywords: antimicrobial activity; cyclic hexapeptide antibiotic; NW-G03; Streptomyces alboflavus 313; structural elucidation
}

In the previous paper, ${ }^{1}$ we described the novel cyclic hexapeptide antibiotic, NW-G01, produced by Streptomyces alboflavus 313. It is structurally related to himastatin ${ }^{2,3}$ and chloptosin, ${ }^{4}$ including a chlorinated pyrroloindoline derivative, but is significantly different in the amino-acid content. In the further study of secondary metabolites of S. alboflavus 313, another novel cyclic hexapeptide NW-G03, a related structural compound of NW-G01, was discovered as a trace constituent from fermentation broth of this strain. The difference of chemical structure between NW-G03 and NW-G01 is only an aminoacid residue. The bioassay results showed that NW-G03 also showed strong antibacterial activity against gram-positive bacteria, including methicillin-resistant Staphylococcus aureus (MRSA), but was ineffective against gram-negative bacteria. NW-G03 also inhibited the growth of three tumor cell lines in vitro.

In this paper, the isolation, structure elucidation, physicochemical properties and in vitro biological activity of compound NW-G03 are described.

NW-G03 was isolated from the fermentation broth of NW-G01producing S. alboflavus 313, as a trace metabolite of interest. As described in our previous paper, ${ }^{1}$ briefly, the supernatant (1001) of the fermentation broth was adsorbed onto a macroporous resin (HPD400, Cangzhou Baoen, Hebei, China), followed by elution with methanol. The elution was evaporated to yield a residue $(60 \mathrm{~g})$. The residue was applied onto a silica gel column and eluted with EtOAc/MeOH (from 100:0 to 0:100). Then the antimicrobial fraction was purified by RP-HPLC (Hypersil $\mathrm{C}_{18}, 20 \mathrm{~mm} \times 250 \mathrm{~mm}, 10 \mu \mathrm{m}$ ) with methanol/water $(75: 25)$ as mobile phase to yield two new compounds NW-G01(168 mg) and NW-G03(11.2 mg). Unfortunately, satisfactory crystals of NW-G03 for X-ray anal could not be obtained.

The physicochemical properties of NW-G03 are summarized in Table 1. It was soluble in dimethyl sulfoxide, methanol and ethyl acetate, but insoluble in water and acetone. It displayed positive color reactions to iodine vapor and argenti nitras solution though it was negative against Molish and $\mathrm{FeCl}_{3}$. The molecular formula of NW-G03 was determined to be $\mathrm{C}_{35} \mathrm{H}_{47} \mathrm{~N}_{10} \mathrm{O}_{7} \mathrm{Cl}(\mathrm{m} / \mathrm{z}$, found $755.3384[\mathrm{M}+\mathrm{H}]^{+}$, calcd 755.3396$)$ on the basis of HR ESI-MS measurement, and the presence of chlorine was suggested by the isotope abundance peaks in the MS spectrum. UV $(\mathrm{MeOH})$ absorptions at $\lambda_{\max } 208 \mathrm{~nm}$ and $\lambda_{\max } 346 \mathrm{~nm}$ indicated the presence of aromatic ring(s) in the molecule.

The ${ }^{13} \mathrm{C}$ and ${ }^{1} \mathrm{H}$ NMR data of NW-G03 are summarized in Table 2. At first, the molecular formula $\mathrm{C}_{35} \mathrm{H}_{47} \mathrm{~N}_{10} \mathrm{O}_{7} \mathrm{Cl}$ was determined by HR ESI-MS (Table 1). As the UV and ${ }^{13} \mathrm{C}$ and ${ }^{1} \mathrm{H}$ NMR spectra of NW-G03 were quite similar to those of NW-G01, the structure of which was determined by single crystal X-ray diffraction. ${ }^{5,6}$ The structure of NW-G03 (Figure 1) was elucidated by comparison with

Table 1 Physico-chemical properties of compound NW-G03

\begin{tabular}{|c|c|}
\hline Properties & Compound NW-GO3 \\
\hline Appearance & White powder \\
\hline m.p. & $193-195^{\circ} \mathrm{C}$ \\
\hline Molecular formula & $\mathrm{C}_{35} \mathrm{H}_{47} \mathrm{~N}_{10} \mathrm{O}_{7} \mathrm{Cl}$ \\
\hline \multicolumn{2}{|l|}{$H R-E S I-M S[m / z]$} \\
\hline Found & $755.3384[\mathrm{M}+\mathrm{H}]^{+}$ \\
\hline Calcd & 755.3396 for $[\mathrm{M}+\mathrm{H}]^{+}$ \\
\hline $\mathrm{UV} \lambda_{\max }^{\mathrm{MeOH}} \mathrm{nm}(\varepsilon)$ & 208 \\
\hline$[\alpha]_{D}^{20}($ c $0.1, \mathrm{MeOH})$ & $-25^{\circ}$ \\
\hline \multicolumn{2}{|l|}{ Solubility } \\
\hline Soluble & DMSO, Methanol, EtOAc \\
\hline Insoluble & $\mathrm{H}_{2} \mathrm{O}$, Acetone \\
\hline \multicolumn{2}{|l|}{ Test } \\
\hline Positive & $\mathrm{I}_{2}, \mathrm{AgNO}_{3}$ \\
\hline Negative & $\mathrm{FeCl}_{3}$, Molish \\
\hline
\end{tabular}

\footnotetext{
${ }^{1}$ State Key Laboratory of Crop Stress Biology in Arid Areas, Northwest A \& F University, Shaanxi, PR China; ${ }^{2}$ Anhui Provincial Laboratory of Agro-Food Safety, Resources \& Environment College, Anhui Agricultural University, Anhui, PR China and ${ }^{3}$ Institutes for Control of Agrochemicals, Shaanxi, PR China

Correspondence: Dr Z Ji or Professor W Wu, Key Laboratory of Plant Protection Resources and Pest Integrated Management, Ministry of Education, College of Plant Protection, Northwest Agricultural \& Forestry University, YangLing, Shaanxi 712100, PR China.

E-mail: jizhiqin@nwsuaf.edu.cn or wuwenjun@nwsuaf.edu.cn

Received 19 February 2010; revised 21 July 2011; accepted 31 August 2011; published online 26 October 2011
} 
Table 2 NMR data of compound NW-G03 and NW-G01 ( $\left.{ }^{1} \mathrm{H} 500 \mathrm{MHz},{ }^{13} \mathrm{C} 125 \mathrm{MHz} \mathrm{CDCl}_{3}\right)$

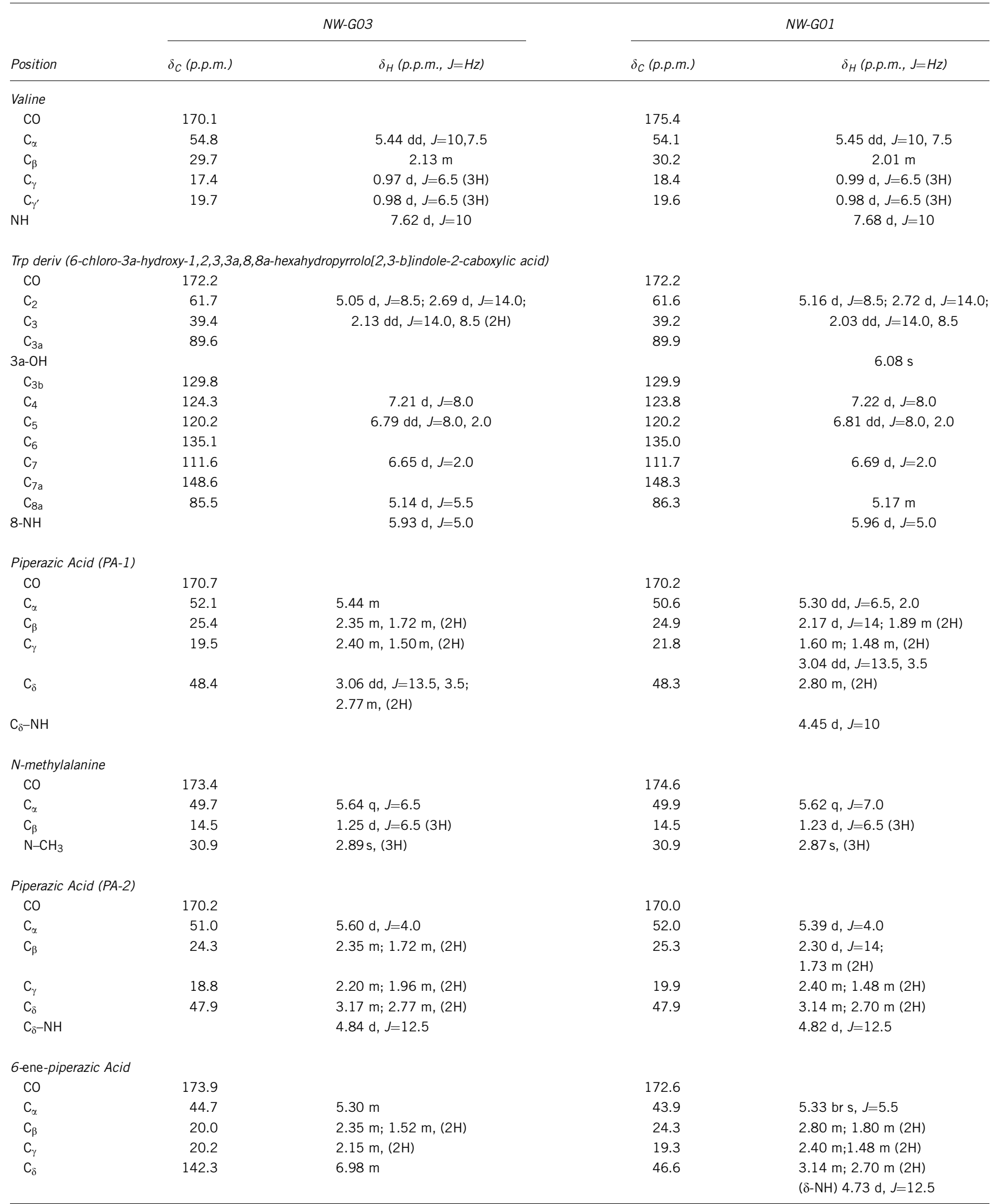




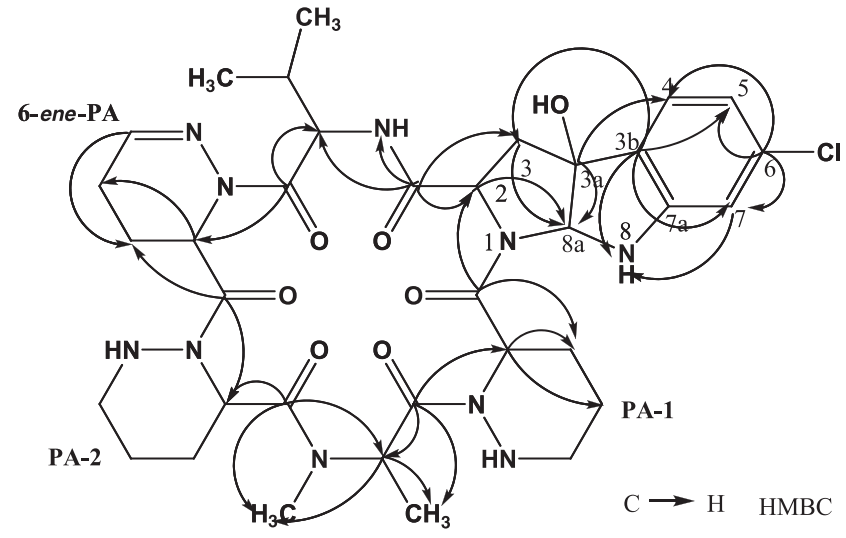

Figure 1 Key HMBC of compound NW-G03. the ${ }^{1} \mathrm{H}$ and ${ }^{13} \mathrm{C}$ NMR data with those of NW-G01 (Table 2), which was finally established by application of a series of 2D NMR techniques. From the ${ }^{1} \mathrm{H}$ and ${ }^{13} \mathrm{C}$ NMR spectra of NW-G03, typical signals for a cyclic peptide were clearly discovered. There were six amide carbonyl carbons $\left(\delta 173.9,173.4,172.2,170.7,170.2\right.$ and 170.1) in the ${ }^{13} \mathrm{C}$ NMR spectrum.

The ${ }^{13} \mathrm{C}$ NMR (DEPT) spectrum showed 35 carbon signals, which were attributed to four methyl carbons, nine methylene carbons, eight methine carbons, three aromatic methine carbon, one $s p^{2}$ methine carbon, one oxygenated quaternary carbon, two quaternary carbons, one chloridated quaternary carbon and six carbonyl carbons by anal of the DEPT and HSQC spectra.

In the ${ }^{1} \mathrm{H}$ NMR spectrum, large coupling $(J=7.5 \mathrm{~Hz})$ were observed between the two $\gamma$ methyl groups at $\delta 0.97,0.98$ and the $\beta$ methine proton at $\delta 2.13$, diagnostic of an isopropyl group. A smaller coupling

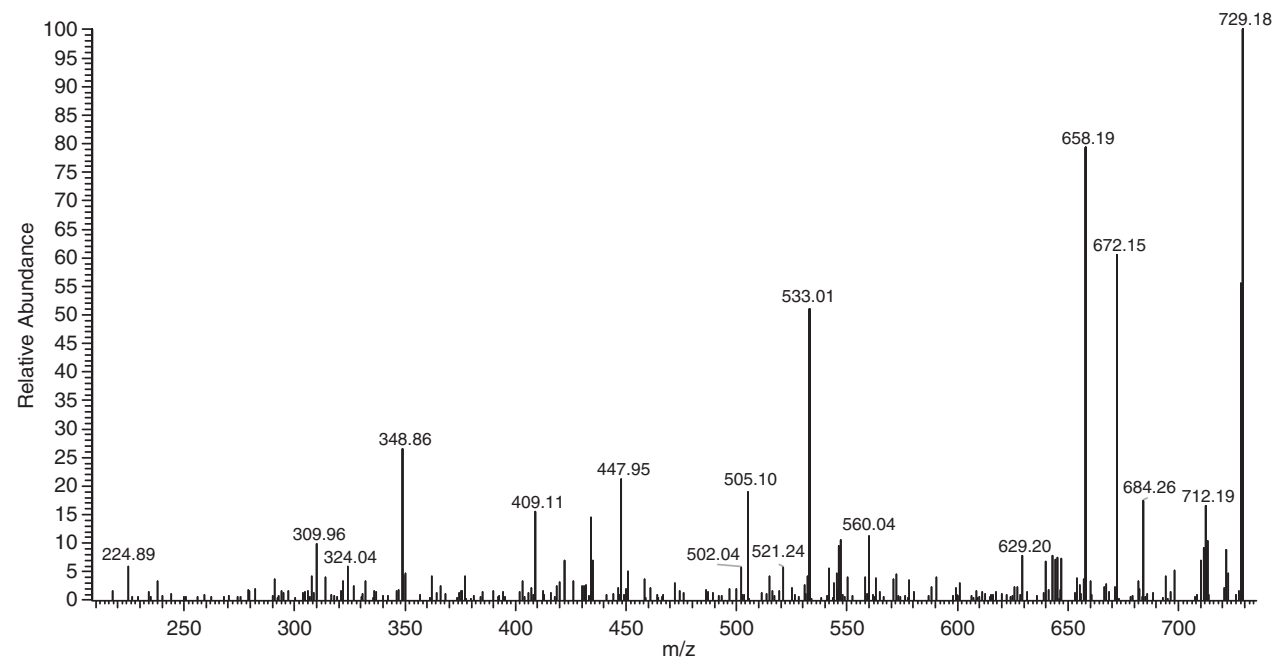

Figure 2 The MS/MS picture of NW-G01 (A mass peak of 739 is missing).

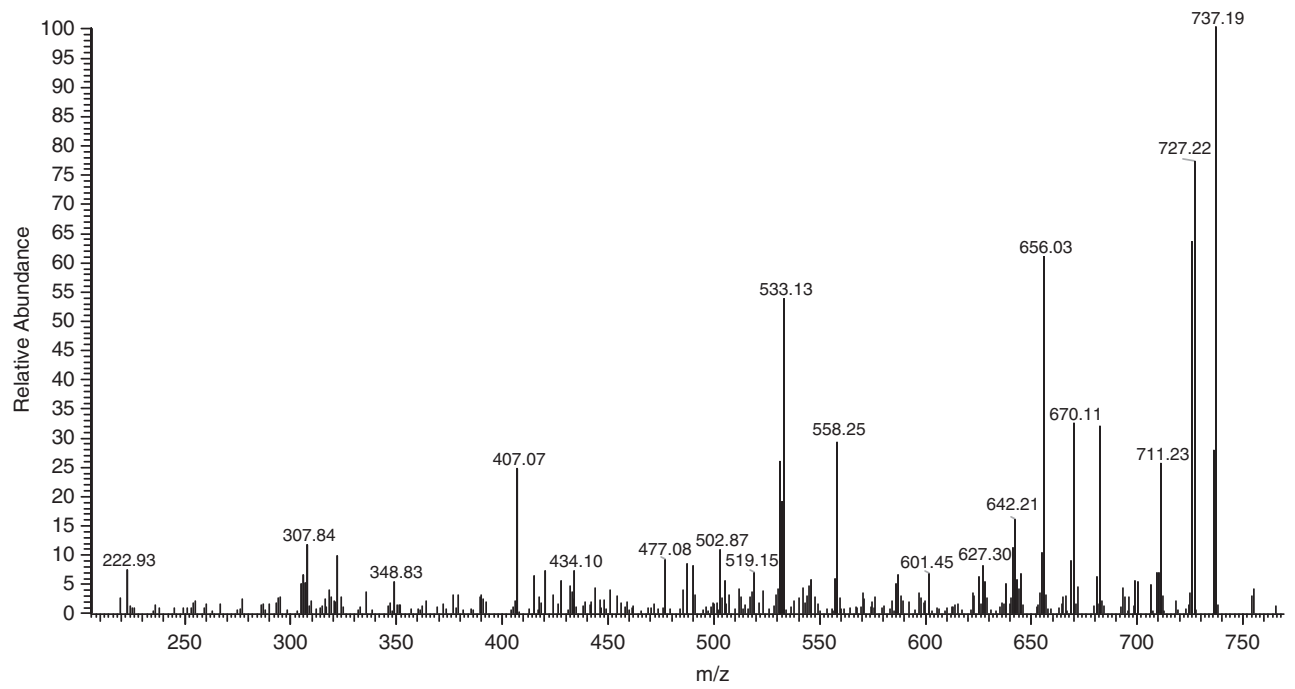

Figure 3 The MS/MS picture of NW-G03. 


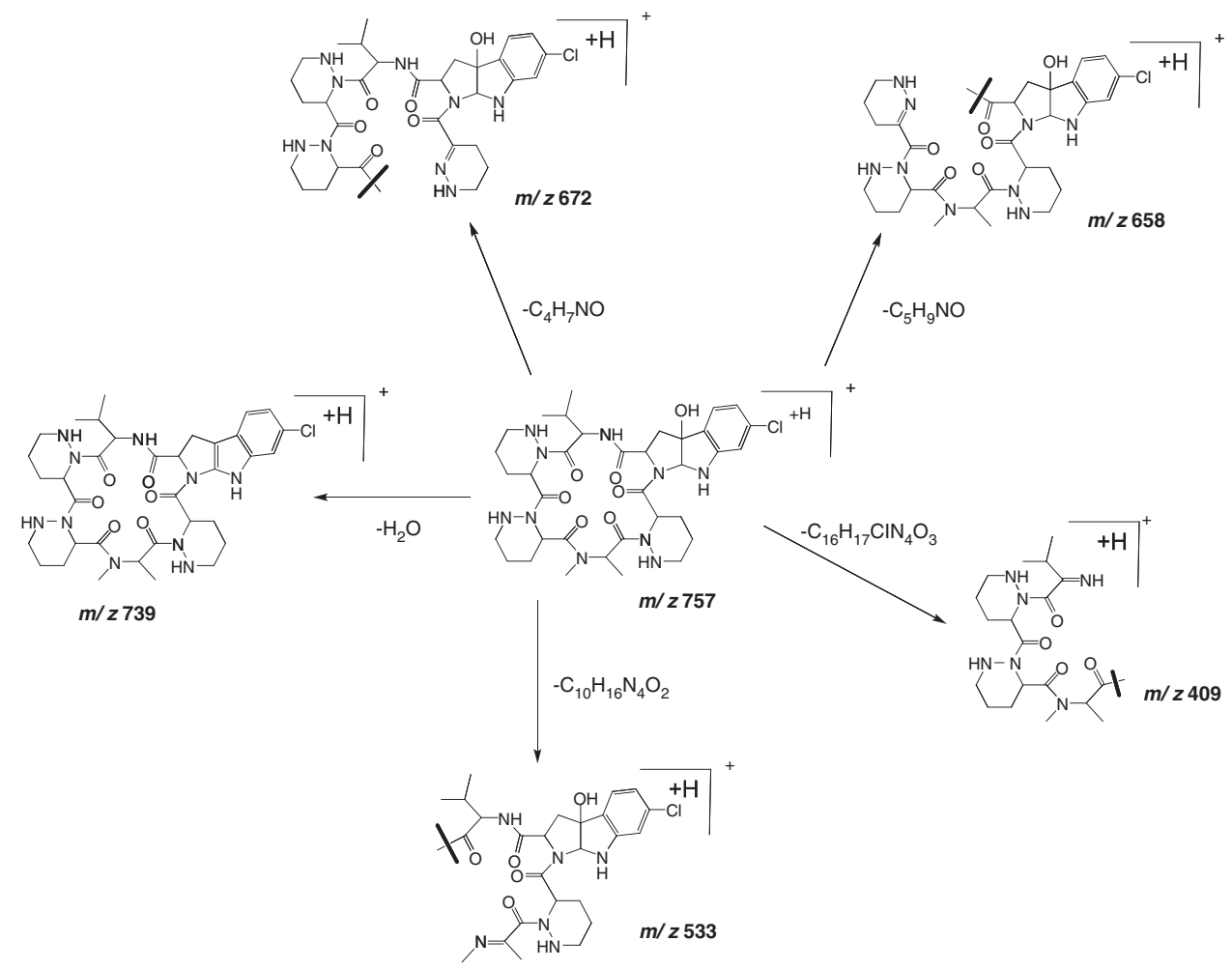

Figure 4 The diagram of the key fragmentations of NW-G01.

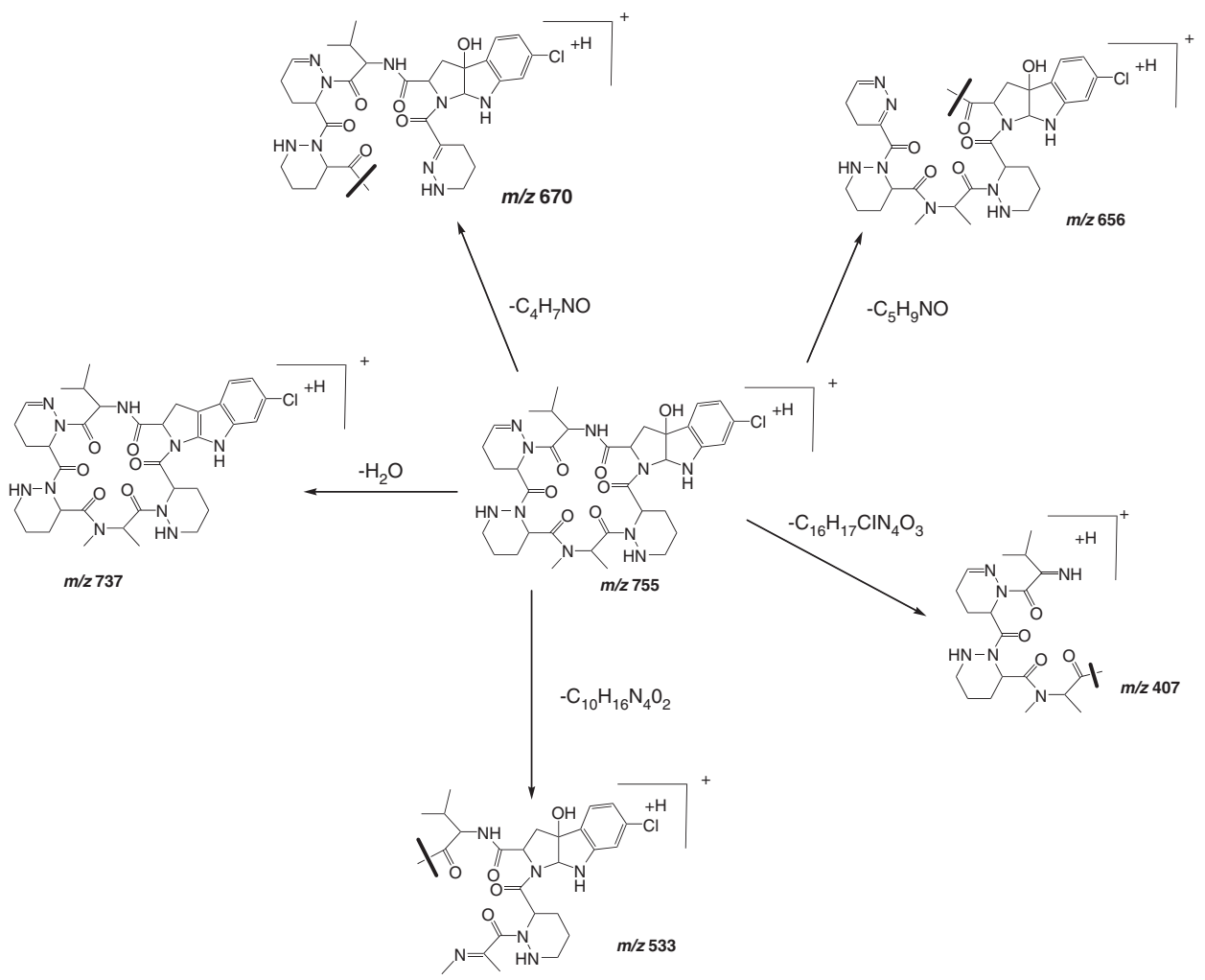

Figure 5 The diagram of the key fragmentations of NW-G03. 
$(J=5.5 \mathrm{~Hz})$ was observed between the $\beta \mathrm{C}-\mathrm{H}(\delta 2.13)$ and $\alpha \mathrm{C}-\mathrm{H}$ $(\delta 5.44)$. A large coupling $(J=10 \mathrm{~Hz})$ between the $\alpha \mathrm{C}-\mathrm{H}(\delta 5.44)$ and the $\mathrm{N}-\mathrm{H}(\delta$ 7.62) completed the spin system. The carbonyl carbon at $\delta 170.1$ showed long-range heteronuclear coupling with $\alpha \mathrm{C}-\mathrm{H}$ $(\delta$ 5.44). The above information indicated the presence of a valine moiety.

According to the same manner described as above, the distinct coupling $(J=6.5 \mathrm{~Hz})$ were displayed between the methyl protons $\left(\delta\right.$ 1.25) and the methine protons $\left(\delta\right.$ 5.64) from ${ }^{1} \mathrm{H}$ NMR spectrum, and the long-range couplings (HMBC) from the methyl single peak protons $(\delta 2.89)$ to the methine carbon $(\delta 49.7)$, and from the methyl protons $(\delta$ 1.25) to a carbonyl carbon $(\delta$ 173.4) diagnosed the presence of a $N$-methylalanine moiety.

Two 3-proton spin systems, including an aromatic ABX system $(\delta 7.21(1 \mathrm{H}, \mathrm{d}, J=8.0 \mathrm{~Hz},) ; 6.79(1 \mathrm{H}, \mathrm{dd}, J=8.0,2.0 \mathrm{~Hz},) ; 6.65(1 \mathrm{H}, \mathrm{d}$, $J=2.0 \mathrm{~Hz}))$, and an aliphatic ABX system $(\delta 5.05(1 \mathrm{H}, \mathrm{d}, J=8.5 \mathrm{~Hz})$; $2.69(1 \mathrm{H}, \mathrm{d}, J=14.0 \mathrm{~Hz},) ; 2.13(1 \mathrm{H}, \mathrm{dd}, J=14.0,8.5 \mathrm{~Hz})$ ) were evident from the ${ }^{1} \mathrm{H}-\mathrm{NMR}$ spectrum. Additionally, other elements of the fragment included an amide proton $(\delta 5.93)$, a quaternary carbon $(\delta$ 89.6), a carbonyl carbon $(\delta$ 172.2) and a chlorine atom. In comparison with spectral data of compound NW-G01, ${ }^{5,6}$ a chlorinated pyrroloindoline derivative(6-chlorl-3a-hydroxy-1,2,3,3a,8,8ahexahydropyrrolo[2,3-b]indole-2-carboxylic acid) was deduced in molecular structure.

In comparison with NW-G01, the remaining residues of NW-G03 included two molecules of piperazic acid (PA) and a molecule of dehydrogenated PA. As only one remaining methenyl carbon $(\delta$ 142.3 ) was present, a $\delta$-dehydro-piperazic acid was diagnosed easily.

To further verify the structural relationship between NW-G01 and NW-G03, The MS/MS experiments of NW-G01 and NW-G03 were carried out and the MS/MS pictures were showed in the Figures 2 and 3, respectively. From the two MS/MS, some main fragment ion peaks were belonged and the fragmentations were determined in the Figures 4 and 5. In the Figures 4 and 5, the NW-G01 fragment ions $(m / z 739,672,658$ and 409) including PA were two more mass units than NW-G03 fragment ions $(\mathrm{m} / \mathrm{z}$ 737, 670, 656 and 407) including PA, furthermore, the fragmentations of NW-G01 and NW-G03 contained the same fragment ion $(\mathrm{m} / z$ 533) without PA part. So, the above results also confirmed that the other structural parts of two compounds were completely uniform except the PA part $(\mathrm{C}=\mathrm{N})$ of NW-G03.

The sequence linkage of the amino-acid residues in NW-G03 was established by the anal of HMBC data, which showed correlation from the $\alpha$-methine protons of amino-acid residue to carbonyl carbon of the neighboring residues (Figure 1).

The absolute configuration of the valine residues was determined by application of the Marfey's method using 1-fluoro-2,4-dinitrophenyl5-L-alanineamide (FDAA) $)^{3,5,7-9}$ and subsequent HPLC anal of the acid hydrolysate of NW-G03. The results of Marfey's anal revealed the presence of D-valine in the acid hydrolysate of NW-G03.

Antibacterial activity was measured by the micro-broth dilution method in 96-well culture plates by employing Mueller-Hinton broth (Hangzhou Microbial Reagent Co. Ltd), according to the Standard of National Committee for Clinical Laboratory (NCCLS). ${ }^{10}$ The compound NW-G03 exhibited significant activity against several grampositive bacteria (Table 3 ) with MIC values of less than $25 \mu \mathrm{g} \mathrm{ml}^{-1}$. No obvious inhibitory effects were observed against gram-negative bacteria at a concentration of $200 \mu \mathrm{g} \mathrm{ml}^{-1}$. The tested gram-positive bacteria were Bacillus cereus 1.1846, Bacillus subtilis 1.88, S. aureus 1.89, MRSA 212 and the gram-negative bacteria were Escherichia coli 1.1636 and Pseudomonas aeruginosa 1.2031.
Table 3 MICs of NW-G03 against the bacteria

\begin{tabular}{lcc}
\hline Name of the tested bacteria & $\begin{array}{c}\text { MIC of } \\
\left.\text { NW-G03( } \mu \mathrm{g} \mathrm{m}^{-1}\right)\end{array}$ & $\begin{array}{c}\text { MIC of Ampicillin } \\
\left(\mu \mathrm{gl}^{-1}\right)\end{array}$ \\
\hline Bacillus cereus (1.1846) & 12.25 & 50 \\
Bacillus subtilis (1.88) & 25 & 50 \\
Staphylococcus aureus (1.89) & 3.07 & 6.13 \\
MRSA (No. 212) & 6.13 & $>100$ \\
Escherichia coli (1.1636) & $>200$ & $>100$ \\
Pseudomonas aeruginosa (1.2031) & $>200$ & $>100$ \\
\hline
\end{tabular}

Table 4 Inhibition of NW-G03 against three tumor cells $\left(30 \mu \mathrm{g} \mathrm{ml}^{-1}\right)$

\begin{tabular}{lcc}
\hline & \multicolumn{2}{c}{ Inhibition rate (\%) } \\
\cline { 2 - 3 } Name of cell lines & NW-G03 & Taxol \\
\hline Bel-7402 & 45.1 & 55.5 \\
HCT116 & 69.3 & 69.5 \\
H460 & 42.0 & 51.1 \\
\hline
\end{tabular}

In addition, antitumor activities of NW-G03 against three human tumor cell lines were tentatively evaluated by the 3-(4,5-dimethyl-2thiazolyl)-2,5-diphenyltetrazolium bromide (MTT) method. ${ }^{11-15}$ As summarized in Table 4, the in vitro compound inhibited the growth of cell lines BEL-7402, HCT-116 and H460 in the concentration of $30 \mu \mathrm{g} \mathrm{ml} \mathrm{m}^{-1}$.

In the isolation process of compound NW-G01, NW-G03, a trace constitutent showed antimicrobial activity was discovered. HR ESIMS measurement exhibited that the molecular formula of NW-G03 only had two less hydrogen atoms than that of NW-G01. Furthermore, ${ }^{1} \mathrm{HNMR}$ and ${ }^{13} \mathrm{CNMR}$ spectral data revealed that these two compounds were highly similar skeleton structures except only one difference that NW-G03 contained a dehydrogenated PA moiety, whereas NW-G01 included a saturated PA moiety. Additionally, the valine residue of NW-G03 was also the D configuration by using Marfery's method, which agreed with the valine absolute configuration of NW-G01.,6 The other residues configurations were confirmed in accordance with compound NW-G01, because the standard substances of these residues were not directly purchased. Therefore, compound NW-G03 is also structurally characterized as an octadeca-membered cyclic hexapeptide composed of D-valine moiety, $\mathrm{N}$-methyl-D-alanine, PAs and a chlorined pyrroloindoline moiety and NW-G03 and NW-G01 were considered as a class of cyclic hexapeptide antibiotics.

In our current study, it was found that NW-G03 had higher antibacterial activities than ampicillin against $B$. cereus 1.1846, B. subtilis $1.88, S$. aureus 1.89 and MRSA 212, especially to MRSA. It was homophilic with antibacterial spectrum of NW-G01, ${ }^{1}$ himastatin $^{7}$ and chloptosin. ${ }^{4}$ Surprisingly, NW-G03 was found to show strong activity against human hepatoma cell line BEL-7402, human colon cancer cell line HCT-116 and human large-cell lung carcinoma cell line H460 cultured in vitro. Further pharmacological studies and an investigation of the mechanism of action are now underway. 


\section{ACKNOWLEDGEMENTS}

This study was supported part by the grant of The National Key Basic Research Program (973 Program, 2010CB126100) from Science and Technology

Ministry of China, Program for New Century Excellent Talents in University from Education Ministry of China and Program for Talents from Northwest A \& F University.

1 Guo, Z Y et al. NW-G01, a novel cyclic hexapeptide antibiotic, produced by Streptomyces alboflavus 313.I.Taxonomy, fermentation, isolation, physico-chemical properties and antibacterial activities. J. Antibiot. 62, 201-205 (2009).

2 Leet, J. E. et al. Himastatin, a new antitumor antibiotic from Streptomyces hygroscopicus III. Structural elucidation. J. Antibiot. 49 (49), 299-311 (1996).

3 Leet, J. E., Schroeder, D. L., Krishnan, B. S. \& Matson, J A Himastatin, a new antitumor antibiotic from Streptomyces hygroscopicus.II. Isolation and characterization. J. Antibiot. 43, 960-961 (1990).

4 Umezawa, K., Ikeda, Y., Uchihata, Y., Naganawa, H \& Kondo, S Chloptosin, an apoptosis-inducing dimeric cyclohexapeptide produced by Streptomyces. J.Org.Chem. 65, 459-463 (2000).

5 Guo, Z. Y. et al. NW-G01, a novel cyclic hexapeptide antibiotic, produced by Streptomyces alboflavus 313.II: Structural elucidation. J. Antibiot. 63, 231-235 (2010).
6 Guo, Z. Y. et al. The correction of 'NW-G01, a novel cyclic hexapeptide antibiotic, produced by Streptomyces alboflavus 313.II: Structural elucidation'. J. Antibiot. 63, 733 (2010).

7 Leet, J. E. et al. Himastatin, a new antitumor antibiotic from Streptomyces hygroscopicus.I. Taxonomy of the producing organism, fermentation and biological activity. J. Antibiot. 43, 956-960 (1990).

8 Sohda, K. et al. YM-216391, a Novel Cytotoxic Cyclic Peptide from Streptomyces nobilis II. Physico-chemical Properties and Structure Elucidation. J. Antibiot. 58, 32-36 (2004).

9 Yoko, N., Teruhito, L. \& Masaki, S. Enantiomeric resolution of amino acids by thin-layer chromatography. J.Mol.Catal.B:Enzym. 12, 105-108 (2001).

10 National Committee for Clinical Laboratory Standards. Methods for Dilution Antimicrobial Susceptibility Tests for Bacteria that Grow Aerobically (Clinical and Laboratory Standards Institute, PA, USA, 2003).

11 Yasuhide, M., Yamada, T., Numata, A. \& Tanaka, R Chaetomugilins, new selectively cytotoxic metabolites, produced by a marine fish-derived chaetomium species. J. Antibiot. 61, 615-622 (2008).

12 Alley, M. C. et al. Feasibility of drug screening with panels of human tumor cell lines using a microculture tetrazolium assay. Cancer. Re. 48, 589-601 (1988).

13 Zhou, J. J. et al. Improved MTT assay for activity of antitumor agents. Chi. J. Pharm. 24, 455-457 (1993).

14 Boros, C. et al. Isolation and identification of the ilcosalides - cyclic peptolides with selective antibiotic and cytotoxic Activities. J. Antibiot. 59, 486-494 (2006).

15 Fang, L. Z. et al. Hypocrellin D, a cytotoxic fungal pigment from fruiting bodies of the ascomycete Shiraia bambusicola. J. Antibiot. 59, 351-354 (2006). 